\title{
A Two-Stage Bayesian Network for Effective Development of Conversational Agent
}

\author{
Jin-Hyuk Hong and Sung-Bae Cho \\ Dept. of Computer Science, Yonsei University, \\ 134 Shinchon-dong, Sudaemoon-ku, Seoul 120-749, Korea \\ hjinh@candy.yonsei.ac.kr, sbcho@cs.yonsei.ac.kr
}

\begin{abstract}
Conversational agent is a system that provides user with proper information and maintains the context of dialogue based on natural language. When experts design the network for conversational agent of a domain, the network is usually very complicated and is hard to be understood. So the simplification of network by separating variables in the domain is helpful to design the conversational agent more efficiently. Composing Bayesian network as two stages, we aim to design conversational agent easily and analyze user's query in detail. Also, by using previous information of dialogue, it is possible to maintain the context of conversation. Actually implementing it for a guide of web pages, we can confirm the usefulness of the proposed architecture for conversational agent.
\end{abstract}

\section{Introduction}

In the age of information, we need to learn a way for finding proper information. Since information providers do not supply a common method or protocol, users cannot help learning several ways [1]. In many publications, it is well known that dialogue is very useful to exchange information and to understand speaker's intention [2]. Conversational agent provides a familiar interface as it does not use keywords or menu but uses natural language dialogue [3]. In order to interpret user's query, conversational agent has to analyze and infer user's intention. There are various techniques for it, and Bayesian network is distinguished one among them [4,5].

If the design of application domain is complex and many variables are mixed in it, inference of user's intention becomes very difficult. It is because designing the network for conversational agent is very complicated in those domains. In this paper, we propose an architecture of conversational agent using Bayesian network in two stages, with which the design of domain is easier than conventional methods and it is possible to infer user's intention in more detail.

\section{Conversational Agent}

Conversational agent is a system that exchanges information between user and agent using natural language dialogue [6,7]. It understands user's intention through conversation and helps user by executing an appropriate action $[4,8]$. Contrary to conventional ways based on menu and keyword, dialogue which is a medium of interaction between human and computer makes it possible to interact more naturally and to include more complicated information than a usual keyword [2]. Therefore 
conversational agent can be an effective user interface in complex system [3]. Table 1 shows the technologies for developing analysis component of conversational agent.

Table 1. Technologies used in designing analysis component

\begin{tabular}{|c|c|c|c|}
\hline Technology & Example Task & Task Complexity & \\
\hline Simple pattern matching & Web search engine & Simple query answering & \multirow[b]{2}{*}{ Easy } \\
\hline Canned script-based model & Game & Simple answer selection & \\
\hline Finite state model & Long-distance dialing & $\begin{array}{l}\text { Restrict flexibility based on } \\
\text { changing state }\end{array}$ & \multirow{3}{*}{ Hard } \\
\hline Frame-based model & Train reservation & $\begin{array}{l}\text { Information collection based } \\
\text { on simple pattern matching }\end{array}$ & \\
\hline Plan-based model & Kitchen design consultant & Dynamic topic changing & \\
\hline
\end{tabular}

Most approaches except plan-based model are general to design conversational agent. They are designed based on simple conversation, so it can be done without much difficulty. For simple task, they are very simple and work well enough because they are based on static process which predefines all possible types and matches one of them. But they do not manage more realistic conversation, such as dynamic topic changing or proceeding dialogue to solve some problems. Sometimes they are easy to make dialogue needlessly long and to repeat same sentence. Also the size of database increases to analyze a complicated query, and information is apt to be duplicated unnecessarily, thereby the performance of the system decreases rapidly if the domain becomes more complex.

Plan-based model is different from previous approaches in considering user's plan and deciding actions to achieve it. Every time partial information is gathered from each queries, and agent predicts user's intention gradually. So it needs dynamic process to deal with it, and it keeps up dialogue until it collects enough information. Plan-based model usually gets better performance than previous approaches, but it costs too much to implement and maintain the system.

With these techniques, conversational agent has been implemented as a guider for web pages [6] and program [4], buying commodity [3], touring [9] and so forth. There are several commercial products such as Nicole of NativeMinds, SmartBot of Artificial Life, Verbot of Virtual Personalities, and so on.

In this paper, we present a plan-based model for designing analysis component of conversational agent to make it more flexible and powerful. Especially we use a modified Bayesian network as an inference engine of analysis component to deal with a dynamic process which is necessary to understand user's intention in query.

\section{Two-Stage Bayesian Network}

\subsection{Inference with Bayesian Network}

The environment of real application of conversational agent is very uncertain [10]. Because users usually do not describe their problems exactly and cannot select one correct sentence for it, in addition, query includes much uncertainty: ellipsis, useless words or duplicated information. Instead, they use several queries, each of which has only a piece of information about user's goal. In spite of these difficulties, conversational agent has to collect information and to decide the meaning of sentence.

Bayesian probabilistic inference is one of the famous models for inference and representation of the environment with insufficient information. The node of Bayesian 
network represents random variable, while the arc represents the dependency between variables $[11,12]$. In order to infer the network, the structure must be designed and the probability distribution must be specified. Usually the structure is designed by expert while the probability distribution is calculated by expert or collected data from the domain. By observing evidence, the probability of each node is computed without excessive computation by Bayesian inference algorithm based on the conditional probability table and independence assumption.

\subsection{Bayesian Network Architecture}

We can extract two kinds of information, topic and sentence pattern from query [6]. Topic is related with words, while the sentence pattern is related with sequence of words. We adopt them as evidences of Bayesian network. If agent is designed by one Bayesian network using words and sentence pattern of query, the network becomes very complicated. Especially dividing them into two-stage Bayesian network, we can accomplish in-depth analysis of query and avoid the complexity occurred in designing the network for large domain. Fig. 1 shows the overall structure of the proposed architecture of conversational agent.

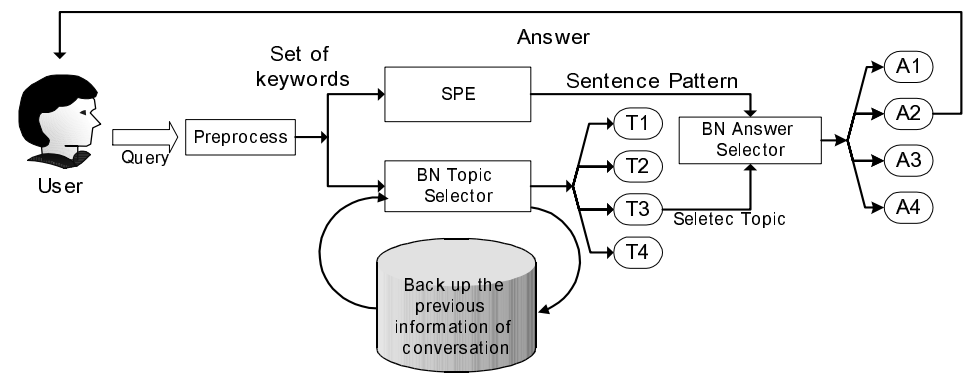

Fig. 1. Structure of the proposed architecture of conversational agent

The proposed system consists of two-stage Bayesian network, BN (Bayesian network) topic selector and BN answer selector, and SPE (Sentence Pattern Extractor) which generates sentence pattern. After user gives a query to conversational agent, it performs preprocessing step to extract predefined keywords. With these extracted keywords BN topic selector executes to infer the topic of the query, and once a topic is selected, BN answer selector corresponding to it obtains a correct answer with sentence pattern generated by SPE. Finally it selects the best answer satisfying the topic and the sentence pattern of query.

\subsubsection{BN Topic Selector}

In this stage we divide the domain into topics that consist of three hierarchical levels by its role, keyword, top-topic and sub-topic. First level is for the keywords used in the domain, and the individuals and attributes of the domain form top-topics. Subtopic represents the individual that has determined its attributes. This hierarchical modeling helps to have the conversation to understand a detailed user's intention [13]. Limiting the scope of common knowledge between human and agent into unit topics, it classifies the intention specifically, and minimizes the uncertainty in each 
hierarchical stage when topic is changed in conversation [13, 14]. It avoids misleading which can be happened by inferring the intention at once, and reduces the uncertainty of inference by progressing hierarchically [9, 10]. Moreover, it gives easy facility of understanding concepts of the domain in designing the network.

When some keywords are included in query, they are used as the evidence of BN topic selector. After inference, we get the highest probability sub-topic as the topic of the query. Fig. 2 shows the process of inference in BN topic selector.

Step 1 : Set the probability of keyword node as 1, whose keyword is included in user's query, and not-included one's as 0 .

Step 2 : Execute Bayesian inference algorithm.

Step 3 : Select the highest probability sub_topic node, above threshold1. If there is no selected node, then go to Step 5.

Step 4 : Select the topic of user's query, and finish the BN topic selector.

Step 5 : Check the top_topic node whose probability is over threshold 2 If there is no node selected, then give up giving an answer. Finish the BN topic selector.

Step 6 : Give an answer of top_topic to collect more information.

Fig. 2. The process of inference of BN topic selector

Usually people do not give just one query to express their intention, and they produce a query based on previous conversation $[15,16]$. Each sentence has a piece of information, so that a query is not sufficient to analyze the topic. By accumulating information through conversation, we get sufficient information to infer the intention. Then we use Bayesian network chain as dynamic knowledge-base to deal with the maintenance of context through conversation. When $\mathrm{BN}$ topic selector infers the topic, it considers network of the last query. By weighing the probability of nodes with previous one, agent uses the piece of information of previous query.

\subsubsection{BN Answer Selector}

Once the topic is selected, BN answer selector corresponding to the selected topic performs to choose an answer that is suitable to the sentence pattern. It uses sentence pattern and detailed keywords of input query as evidences. In this paper, we construct SPE with a set of classifying automata [6]. Sentence patterns can be categorized as shown in Table 2. Classifying automata is designed for each sentence pattern with a set of keywords and sequences, and Fig. 3 shows one for "WhatIf" question.

Table 2. Sentence Pattern

\begin{tabular}{l|l}
\hline Query Classification & Sentence Pattern \\
\hline Question & $\begin{array}{l}\text { Can, Who, WhatIf, Method, Location, Reason, } \\
\text { Should, Time, Description, Fact }\end{array}$ \\
\hline Statement & Message, Act, Is, Have, Want, Fact \\
\hline
\end{tabular}




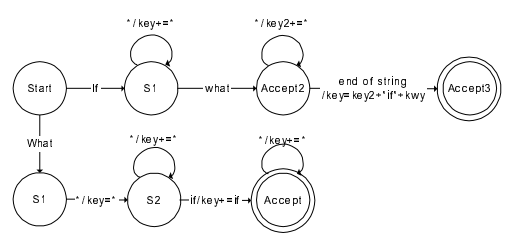

Fig. 3. Automaton for classification of "WhatIf" question

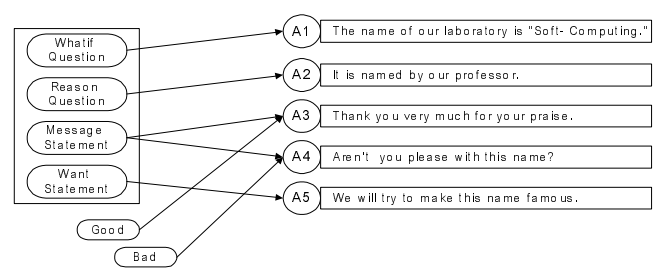

Fig. 4. The structure of BN Answer Selector for "The name of laboratory"

With this sentence pattern generated, BN answer selector selects a proper answer. Each BN answer selector has a set of answers of the topic based on sentence patterns and some detailed keywords. The detailed keywords used in this stage are different from those used in BN topic selector in detailed level. Fig. 4 shows the structure of BN answer selector for topic, "The name of laboratory."

\section{Illustration}

In this paper, a query is divided into three types: 1) Enough-information, 2) useprevious-information, and 3) need-more-information. That is because in common conversation an ellipsis is frequently happened, and usually speaker uses background knowledge. The proposed conversational agent can deal with these types of queries.

\subsection{Enough-Information Type}

If user's query contains all the information to estimate user's intention, agent gives a proper answer obtained by the inference of the system. Dialogue 1 is an example for this case.

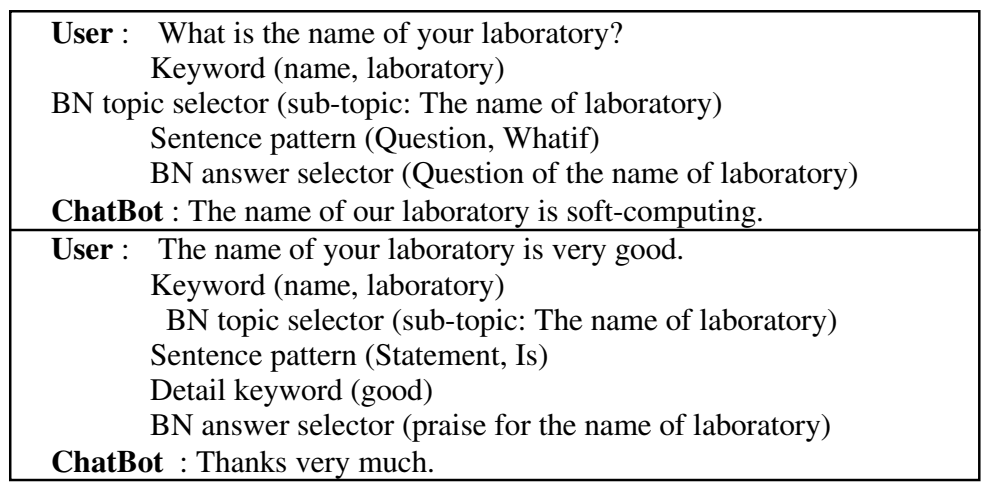

Dialogue 1. Enough-information type and differentiation by sentence pattern 


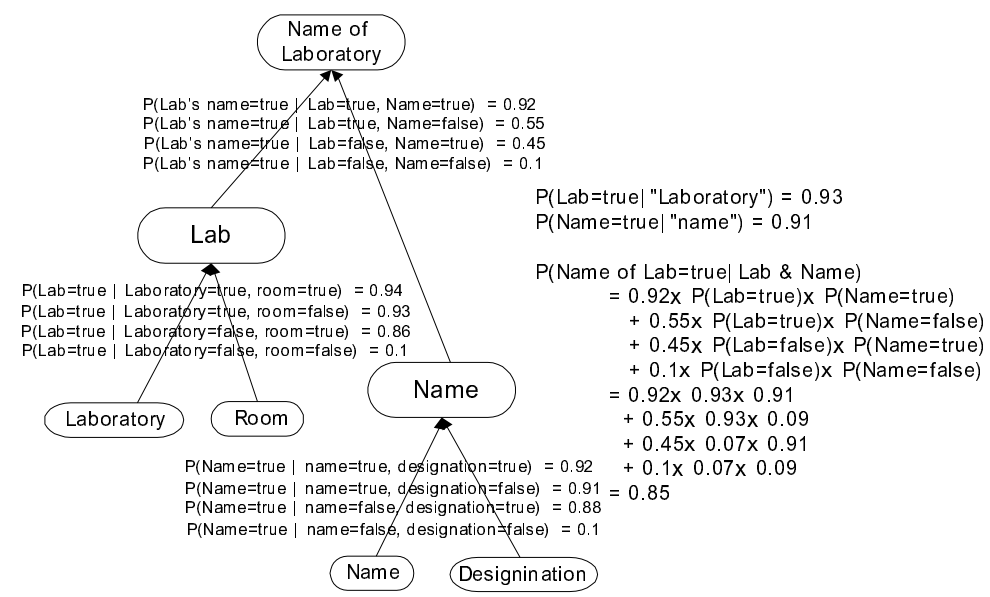

Fig. 5. Simple Bayesian network to show the course for inference of "Name of Lab"

In the preprocessing step, the words "laboratory" and "name" are extracted, they are passed as inputs of $\mathrm{BN}$ topic selector. $\mathrm{BN}$ topic selector executes the inference with them as evidences, picks up the best suitable topic for user's query. Fig. 5 shows the minimum Bayesian network for selecting the topic, "Name of Lab." First, the probability of node for extracted keyword is set as 1 and the others are set as 0 . With Bayesian inference algorithm, the values of node "Name" and "Lab" become 0.91 and 0.93, respectively. The probability of sub-topic "Name of Lab" becomes 0.85 which exceeds the threshold of 0.75 , and is selected as the topic of user's query. Usually the others are not selected because they may not go beyond the threshold, otherwise the highest one is selected among them. Consequently BN answer selector for "Name of Lab" is activated. With the WhatIf-question type obtained by SPE, it understands that user wants to know the name of laboratory and answers about that. It is possible that the real meaning of the queries is different while the topics of queries are same, because of sentence patterns. That is as shown in lower part of Dialogue 1.

\subsection{Use-Previous-Information Type: Maintain the Context of Conversation}

Through the conversation between human and agent, user may change the topic of what he or she wants to know. In this case, agent has to maintain the context of conversation [16]. Because most of topic changed is based on previous dialogue, the content of previous dialogue provides the information to analyze the current dialogue. Dialogue 2 is the example dialogue for this case.

\subsection{Need-More-Information Type: Active Conversation}

In many cases user has background knowledge in addition to the contents of conversation, so that it frequently happens that the query does not include all the information for the inference of user's intention. It is because the knowledge bases for user and agent are different. The agent manages to infer the topic of user's query roughly, and the supplementary information must be supplied. Agent gets this supplementary information by requesting to user. When the agent asks to user, 
it bases a top-topic that is roughly inferred. Dialogue 3 is the example of active conversation of agent.

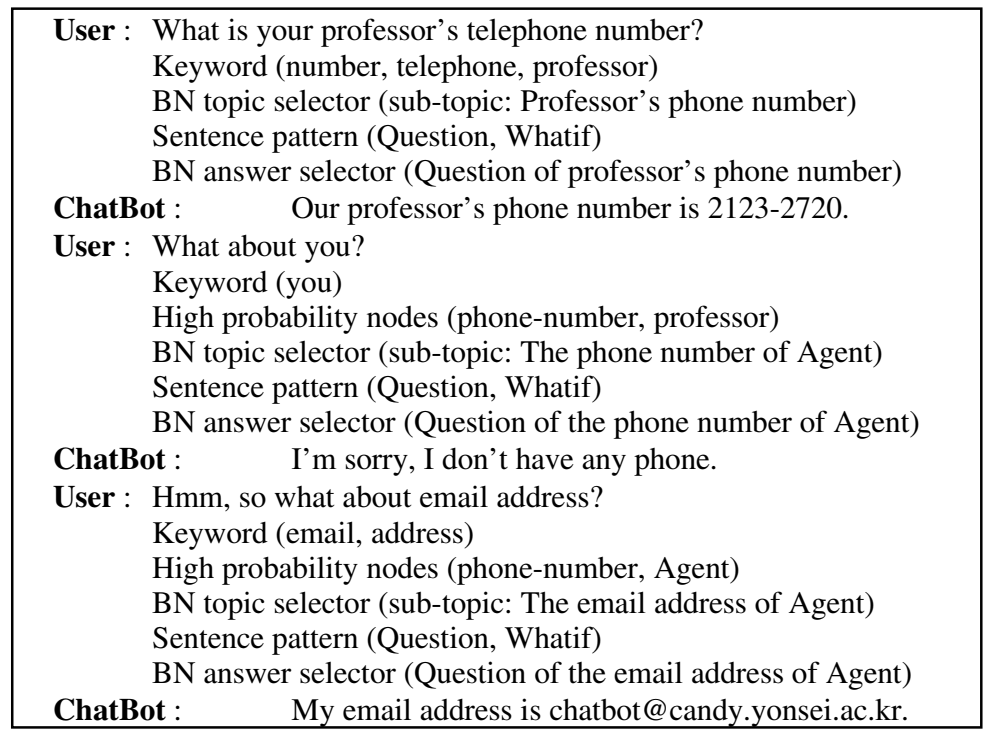

Dialogue 2. Use-previous-information Type

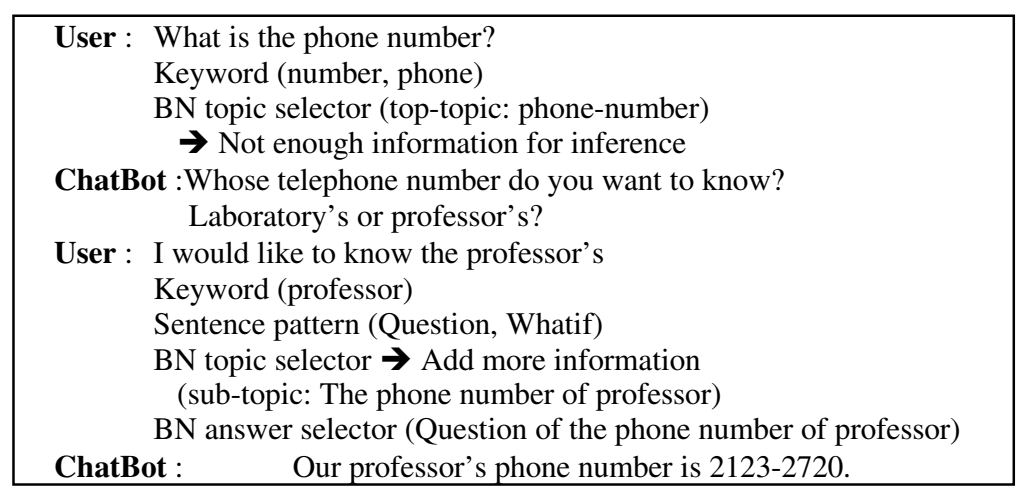

Dialogue 3. Need-more-information Type

\section{Concluding Remarks}

Conversation is one of the most representative communication methods to express one's knowledge and intention. However it has uncertainty, such as implication and ellipsis, and it usually uses one's background knowledge. So there are limits with conventional approaches like simple pattern matching to manage them. Moreover if the domain becomes larger, it becomes very hard to design each pattern or network. 
In this paper, we model conversational agent using two-stage Bayesian network, with which it gets to be more flexible and detailed to infer the intention. Besides, the task of designing network becomes easier and more comprehensible by one's intuition. We analyze the conversation on the types of queries to induce more active conversation. If some information is insufficient to infer, agent can ask user more information. Using sentence pattern, more suitable type of answer is offered. Bayesian network chain is useful to maintain the context of conversation.

Analysis of domain and design of network is prerequisite to develop conversational agent. They are dependent on human's hand, so that it is very expensive. As the application domain changes, the network must be changed. The research on automatic construction of network is left for future work, in order to let the human design abstract network and construct detailed networks automatically. Some problems commonly occurred in conversation, such as social interaction and insertion sequence, will be solved out with future work.

Acknowledgements. This paper was supported by Biometrics Engineering Research Center, and Brain Science and Engineering Research Program sponsored by Korean Ministry of Science and Technology.

\section{References}

1. Y. Yang, L. Chien and L. Lee, "Speaker intention modeling for large vocabulary mandarin spoken dialogues," Proc. of the 4th Int. Conf. on Spoken Language, pp. 713-716, 1996.

2. J. Allen, D. Byron, M. Dzikovska, G. Ferguson, L. Galescu and A. Stent, "Towards conversational human-computer interaction," AI Magazine, 22(4), pp. 27-37, 2001.

3. J. Chai, V. Horvath, N. Nicolov, M. Budzikowska, N. Kambhatla, and W. Zadrozny, "Natural language sales assistant: A web-based dialog system for online sales," Proc. of the 13th Annual Conf. on Innovative Applications of Artificial Intelligence, pp.19-26, 2001.

4. E. Horvitz, J. Breese, D. Heckerman, D. Hovel and K. Rommelse, "The lumiere project: Bayesian user modeling for inferring the goals and needs of software users," Proc. of the 14th Conf. Uncertainty in Artificial Intelligence, pp. 256-265, 1998.

5. D. Albrecht, I. Zukerman, A. Nicholcon and A. Bud, "Towards a Bayesian model for keyhole plan recognition in large domains," Proc. of the 6th Int. Conf. on User Modeling, pp. 365-376, 1997.

6. S.-I. Lee, C. Sung and S.-B. Cho, "An effective conversational agent with user modeling based on Bayesian network," Lecture Notes in Computer Science, 2198, pp. 428-432, 2001.

7. S. Macskassy and S. Stevenson, "A conversational agent," Master Essay, Rutgers University, 1996.

8. D. Heckerman and E. Horvitz, "Inferring informational goals from free-text queries: A Bayesian approach," Proc. of the 14th Conf. on Uncertainty in Artificial Intelligence, pp.230-237, 1998.

9. G. Ferguson, J. Allen and B. Miller, "TRAINS-95: Towards a mixed-initiative planning assistant," Proc. of the 3rd Conf. on Artificial Intelligence Planning Systems, pp.70-77, 1996.

10. T. Paek and E. Horvitz, "Conversation as action under uncertainty," Proc. of the 16th Conf. on Uncertainty in Artificial Intelligence, pp.455-464, 2000.

11. E. Charniak, "Bayesian networks without tears," AI Magazine, 12(4), pp.50-63, 1991. 
12. T. Stephenson, "An introduction to Bayesian network theory and usage," IDIAP-RR0003, 2000.

13. E. Horvitz and T. Paek, "A computational architecture for conversation," Proc. of the 7th Int. Conf. on User Modeling, pp.201-210, 1999.

14. J. Allen, G. Ferguson and A. Stent, "An architecture for more realistic conversational systems," Proc. of Intelligent User Interfaces, pp.1-8, 2001.

15. J. Allen, "Mixed Initiative Interaction," IEEE Intelligent Systems, 14(6), pp.14-23, 1999.

16. X. Wu, F.Zheng and M. Xu, "TOPIC Forest: A plan-based dialog management structure," Int. Conf. on Acoustics, Speech and Signal Processing, pp.617-620, 2001. 